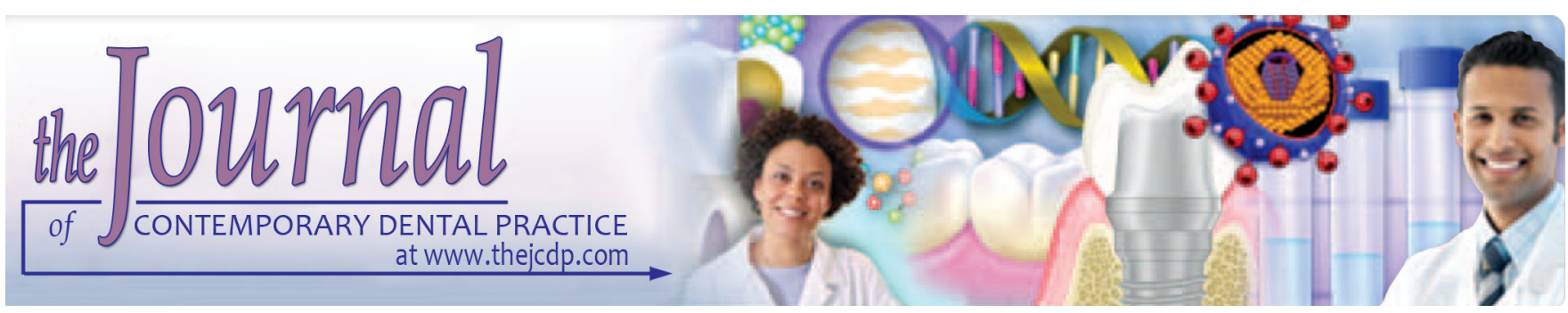

\title{
Fibrinogen Demonstration in Oral Lichen Planus: An Immunofluorescence Study on Archival Tissues
}

\author{
${ }^{1}$ Pallavi D Shirol, ${ }^{2}$ Veena Naik, ${ }^{3}$ Alka Kale
}

\begin{abstract}
Background: Lichen planus is a premalignant condition with minimal diagnostic aids. This study is an attempt to use paraffin embedded sections of lichen planus with immunofluorescein stain and to evaluate the immunofluorescent sections to establish pattern of fibrinogen deposition.
\end{abstract}

Materials and methods: Thirty-five paraffin embedded sections of old and new cases of oral lichen planus (study group) and five normal oral mucosa (control group) were chosen. Two sections of each $(H \& E)$ case were taken, one was stained with hematoxylin and eosin and another with fluorescein isothiocynate conjugate (FITC) polyclonal rabbit antibody against fibrinogen. Fluorescent findings were examined with a fluorescent microscope.

Results: A high statistical significant correlation was found in respect to fluorescence positivity, intensity of fluorescence and distribution of fluorescence each with $p<0.0001$ and fluorescence at blood vessel walls $(p=0.0003)$.

Conclusion: This study suggested that paraffin embedded sections can be successfully used in direct immunofluorescence staining in routine set up where only formalin fixed tissues are received.

Clinical significance: Paraffin embedded sections can be successfully used in direct immunofluorescence staining when only formalin fixed tissues are received.

Keywords: Direct immunofluorescence, Fibrinogen, Oral lichen planus, Paraffin sections.

How to cite this article: Shirol PD, Naik V, Kale A. Fibrinogen Demonstration in Oral Lichen Planus: An Immunofluorescence Study on Archival Tissues. J Contemp Dent Pract 2015;16(10): 824-828.

${ }^{1}$ Department of Oral Pathology, MA Rangoonwala College of Dental Sciences \& Research Centre, Pune, Maharashtra, India

${ }^{2,3}$ Department of Oral Pathology, KLES Institute of Dental Sciences, Belgaum, Karnataka, India

Corresponding Author: Pallavi D Shirol, Reader, Department of Oral Pathology, MA Rangoonwala College of Dental Sciences \& Research Centre, Pune, Maharashtra, India Phone: 9970133664, e-mail: pdshirol@yahoo.com

\section{Source of support: Nil}

Conflict of interest: None

INTRODUCTION

Lichen planus, a term first described by Erasmus Wilson in 1869 , is a fairly common chronic inflammatory skin disease, which often affects the oral mucosa. It could be associated with stress, malnutrition, infection or local trauma, immune reactions and autoimmunity. ${ }^{1}$

Many lesions of oral mucosa are difficult to diagnose on clinical grounds alone and conventional histopathologic examination may be inconclusive. Immunofluorescence microscopy is a valuable adjunct in the diagnosis of oral mucosal diseases, including lupus erythematosus, pemphigus vulgaris, mucous membrane pemphigoid, erythema multiforme and lichen planus. ${ }^{2}$

Despite much recent research, the etiology and pathogenesis of lichen planus remains undetermined. Immunofluorescent studies in lichen planus lesions have provided some insight into a proposed immunopathogenesis..$^{1-3}$

Immunofluorescence studies on frozen sections of oral lichen planus demonstrates fibrinogen deposition in basement membrane zone, within colloid bodies and in the blood vessel walls. ${ }^{3-6}$

Although oral lichen planus has an immune pathogenesis, studies dealing with immunopathology on paraffin embedded tissue sections of oral lichen planus have not been studied and reported extensively.

Immunofluorescent technique has been diagnostic in vesiculobullous lesions, especially when clinical and histopathology is not so. Retrospective evaluation on routine histological material do not seem possible as specific and reliable immunolabeling is thought to require fresh frozen biopsy specimens to yield the best results. 
But in routine clinical and diagnostic laboratories and at institutional level, the feasibility of these may not be available. Secondly, for retrospective studies and for follow-up cases, repeat biopsy for the frozen sections may be unethical.

This study aims in presenting a simple, economic and effective technique for diagnostic immunofluorescence testing on archival histological material from oral biopsies of lichen planus.

\section{MATERIALS AND METHODS}

This study included 35 cases of lichen planus as study group and five normal mucosa as controls. Cases were obtained from the archivals of department of oral pathology. The study group consisted of 35 clinically diagnosed and histologically confirmed cases of lichen planus.

Criteria for clinical diagnosis were presence of bilateral lesions, reticular pattern along with other pattern. Criteria for histological diagnosis were presence of basal cell degeneration and a band of lymphocytes in the juxtaepithelial region.

Formalin fixed paraffin embedded tissues of the same were retrieved from the archives of department of oral pathology, for the purpose of immunofluorescence study. Two sections of $5 \mu$ thickness were taken. One section was stained for routine $\mathrm{H} \& \mathrm{E}$ and other was taken for immunofluorescent staining.

Before immunofluorescent staining, the dewaxed and rehydrated sections were rinsed in two changes of phosphate buffered saline (PBS I and II) which was used as wash buffer for 15 minutes each. Heat induced epitope retrieval (HIER): A glass couplin jar containing EDTA buffer was placed in pressure cooker with water at its base. Lid was closed and buffer was allowed to

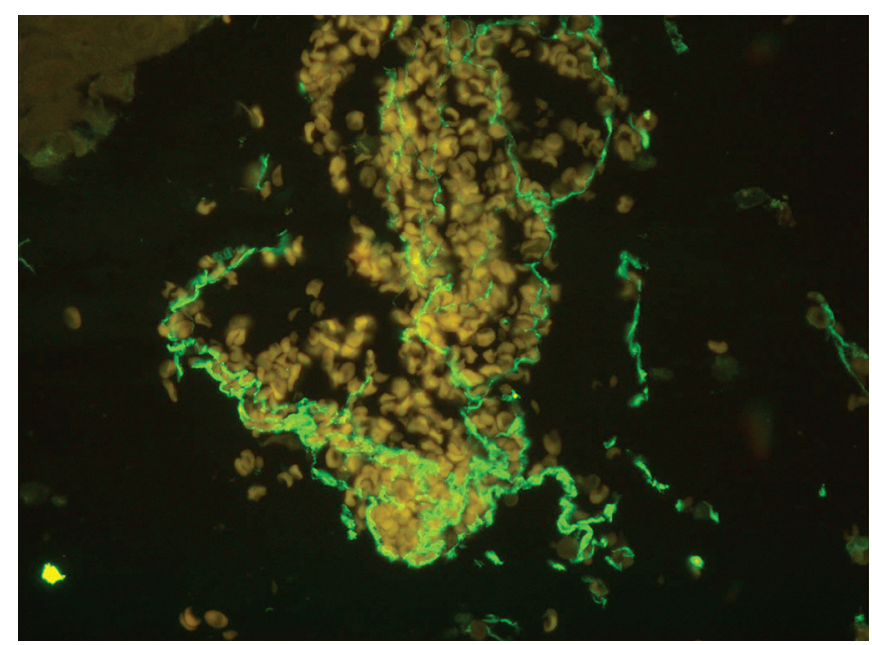

Fig. 1: Photomicrograph showing fibrin meshwork in the extravasated RBCs (positive control) boil. After this, the lid was opened and slides were kept in the couplin jar containing the buffer. Lid was closed; pressure was built up for 5 minutes. The couplin jar was removed and was allowed to cool. After the slides were cooled, slides were passed through PBS III and PBS IV for 15 minutes each. Slides were incubated with 1:50 dilution of Fluoroscene isothiocyanate (FITC) labeled rabbit antisera against fibrinogen (DAKO) in PBS in wet humidifying chamber at room temperature for an hour. Slides were taken out, excess was drained and were washed in PBS V for 5 minutes. Slides were dried around the sections and were mounted with glycerol, coverslip was placed and were observed immediately.

Blue filter in the range of 460 to $490 \mathrm{~nm}$ was used which showed apple green fluorescence. Evaluation was based on the apple green fluorescence at the basement membrane zone. Fibrin in the extravasated RBC's and within the blood vessels of the tissue sections was considered as positive control (Fig. 1). The data collected was statistically analyzed with Chi-square test.

\section{RESULTS}

The most important criterion for a positive diagnosis was the presence of fluorescence at dermoepidermal junction.

Among 35 cases, the positive fluorescence at the dermoepidermal junction was observed in 34 cases. Statistical evaluation revealed a highly significant fluorescence positivity, with $p<0.0001$. The normal oral mucosa cases did not show any fluorescence (Fig. 2). The pattern of fluorescence was linear deposition with or without threadlike extensions into superficial connective tissue (Figs 3 and 4). Among 35 cases, 30 cases showed fluorescence at the blood vessel wall in the superficial connective tissue (Fig. 5), with high statistical significance $(\mathrm{p}=0.0003)$ (Tables 1 and 2).

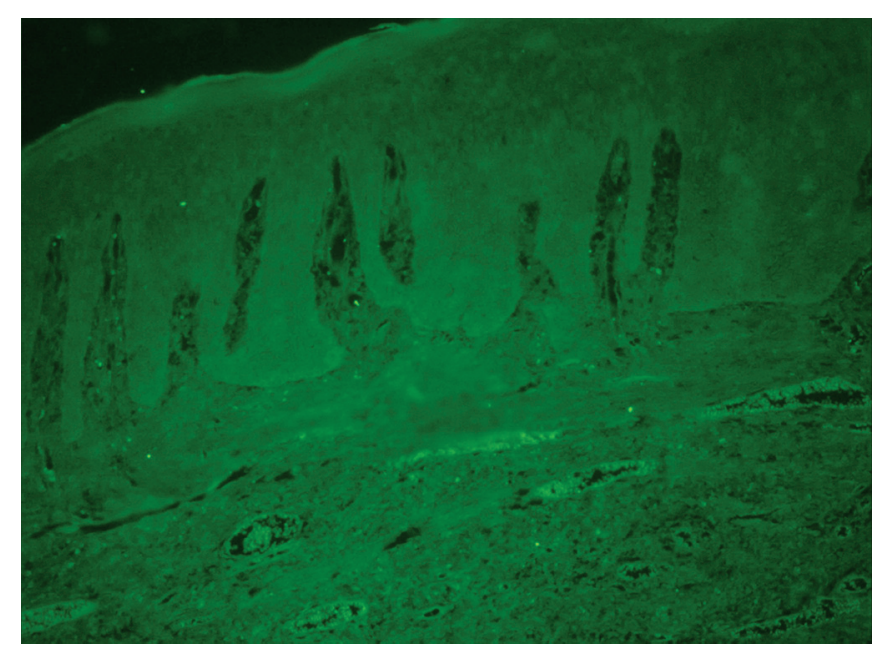

Fig. 2: Photomicrograph showing normal oral mucosa showing absence of fluorescence 


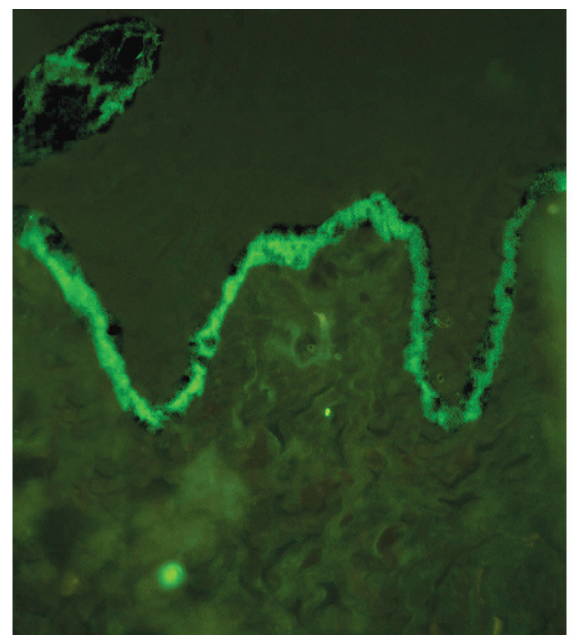

Fig. 3: Photomicrograph showing fluorescence at dermoepidermal junction

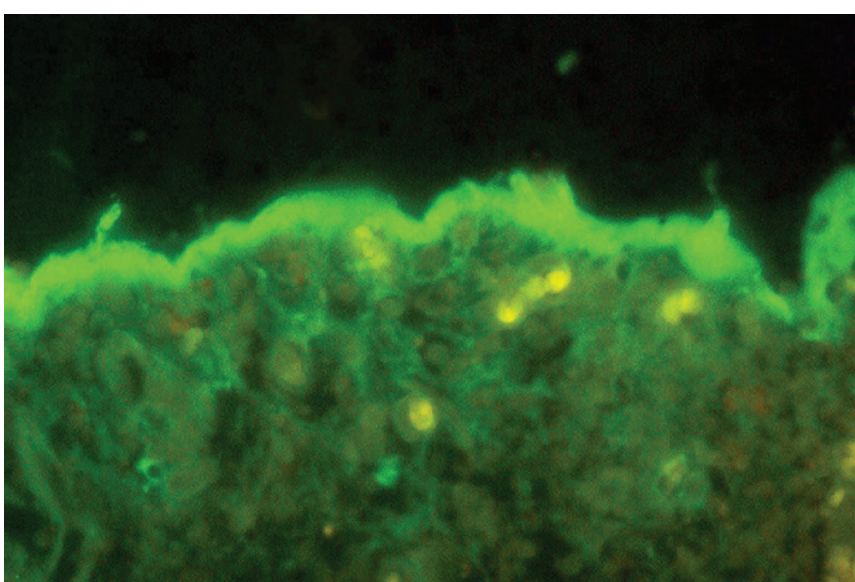

Fig. 4: Photomicrograph showing fluorescence at dermoepidermal junction with thread like extensions

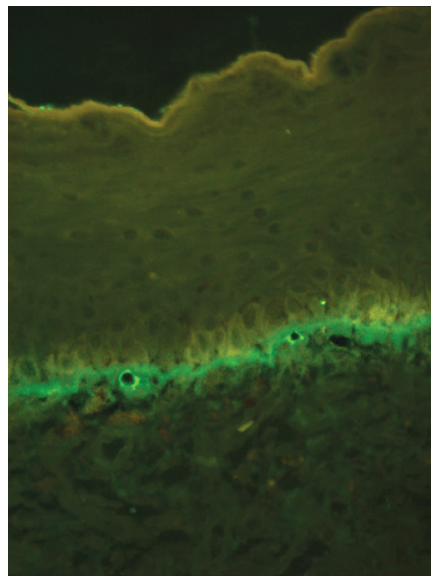

Fig. 5: Photomicrograph showing fluorescence at dermoepidermal junction with fluorescence around blood vessel

Table 1: Fluorescence positivity: among 35 cases, $97.14 \%$ showed fluorescence positivity and statistical analysis was highly significant $(p<0.0001)$

\begin{tabular}{llllll}
\hline & \multicolumn{2}{c}{ Study group } & & \multicolumn{2}{c}{ Control group } \\
\cline { 2 - 3 } \cline { 5 - 6 } Fluorescence & Total & Percentage & & Total & Percentage \\
\hline Positive & 34 & 97.14 & & 0 & 0 \\
Negative & 01 & 2.86 & & 5 & 100 \\
\hline
\end{tabular}

Table 2: Fluorescence at blood vessel wall: Out of 35 cases, $85.71 \%$ showed fluorescence at the blood vessel wall and this finding showed high statistical significance with $p=0.0003$

\begin{tabular}{llllll}
\hline \multirow{2}{*}{$\begin{array}{l}\text { At blood vessel } \\
\text { wall }\end{array}$} & \multicolumn{2}{c}{ Study group } & & \multicolumn{2}{c}{ Control group } \\
\cline { 2 - 3 } \cline { 5 - 6 } & Total & Percentage & & Total & Percentage \\
\hline Positive & 30 & 85.71 & & 0 & 0 \\
Negative & 05 & 14.29 & & 5 & 100 \\
\hline
\end{tabular}

\section{DISCUSSION}

Lichen planus is a fairly common chronic inflammatory skin disease which often affects the oral mucosa without skin manifestations. Despite much recent research, the etiology and pathogenesis of the disease remains undetermined.

Immunofluorescent studies in lichen planus lesions have provided some insight into a proposed immunopathogenesis. In cases where clinical and histopathologic evaluation cannot confirm the diagnosis satisfactorily, the use of direct immunofluorescence is of great importance.

A number of immunofluorescent studies in lichen planus on frozen sections have been reported till date. But in routine setup it is difficult to avail fresh frozen sections. Hence in this study, paraffin embedded tissue sections of lichen planus were utilized for direct immunofluorescence study to demonstrate fibrinogen.

Among 35 cases, 34 cases showed positive fluorescence at the dermoepidermal junction and in 30 cases at blood vessel wall. No significant immunofluorescence staining was observed in normal oral mucosal biopsies. Fluorescence positivity showed high statistical significance $(\mathrm{p}<0.0001)$.

Out of 35 cases studied, 34 showed fibrinogen deposition at basement membrane zone $(p<0.0001)$ and 30 cases showed fibrin at small blood vessel along with basement membrane zone ( $p=0.0003)$. Findings of our study showed high statistical significance.

It is suggested that immunofluorescent changes in lichen planus are secondary events, consequences of damage to the lower epidermis and basement membrane zone. Fibrin is laid down and inflammatory cells move over it and release cytokines and interleukins. The explanation for fibrin deposition at blood vessel wall, could be due to the local secretion of cytokines by mononuclear inflammatory cells leading to increased microvascular permeability, in turn giving rise to dermal edema and fibrin deposition. ${ }^{7,8}$ This also explains the expression of fibrinogen at blood vessel walls in the superficial part of connective tissue in lichen planus.

Abell E, Presbury DGC, Marks R and Ramnarain D et al in their study on frozen sections found that fibrin was deposited evenly against the epidermo-dermal junction. 
Occasionally producing a thin homogenous band, but more often irregular strands extended from the junctional band into the papillary or parafollicular dermis. Similar findings were observed in our study. The fibrin staining frequently demonstrated the colloid body deposits in both the epidermis and dermis and occasionally fibrin was detected in and around blood vessels of papillary connective tissue. $^{9}$

Daniels and Quadra-White in their study on frozen sections found that out of 31 specimens, that reacted with anti-firbinogen 30 showed intense positive fluorescence that outlined the basement membrane zone and had numerous irregular extensions into the superficial lamina propria. ${ }^{10}$

Laskaris et al in their study on frozen sections found that out of 35 cases, 34 cases showed fibrin deposition at the basement membrane zone either in a linear pattern or more often in a broad band from which numerous threadlike outgrowths extended into the corium and to a minor degree into the epithelium. Fibrin deposition within colloid bodies was seen in 14 cases and around blood vessel walls in 5 cases. Similar patterns of fibrinogen deposition were found in our study. ${ }^{1}$

In our study out of 35 cases, 34 showed florescence at the dermal-epidermal junction, showing linear pattern with extensions into the connective tissue. This finding was in concordance with previous studies. ${ }^{9,10}$ Fibrin deposition was not seen in cytoid bodies in our study.

Fluorescence positivity at basement membrane zone seen in our study also was in concordance with study by Raghu et al. ${ }^{5}$

Firth et al in their study on comparison of fresh frozen tissue and formalin fixed paraffin embedded tissue, found that the pattern of staining in cases of lichen planus was essentially a fibrillar pattern at the basement membrane zone. They also found that intensity of staining was less than that obtained from using fresh frozen tissue. ${ }^{3}$

Kolde et al conducted direct immunofluorescence testing on archival histological material from oral biopsies. The study was performed on 30 biopsies of suspected lichen planus. Immunofluorescence examination showed excellent preservation of tissue morphology, and negligible background staining, nonspecific fluorescence and tissue autofluorescence. They concluded saying that immunofluorescence testing on pronase treated histological sections is a valuable diagnostic tool that improves the often controversial histopathological assessment of oral lichen planus. ${ }^{2}$

In our study, out of 35 cases, one case did not show any significant fluorescence, but $\mathrm{H} \& \mathrm{E}$ stained section of same case showed basal cell degeneration and inflammatory component juxtaepithelially. This tissue was stained twice for immunofluorescence, both the times it was negative. The reason for negativity could not be established, could be either due to fixation or processing errors.

Authors suggested that this pattern of fibrinogen deposition in the absence of fluorescence by other reagents is sufficiently unique to be used as a diagnostic criterion for oral mucosal lichen planus. ${ }^{11,12}$

In many cases, the fixation and processing steps involved in the preparation of tissues results in loss of immunoreactivity of antigens. This can be reversed by the use of appropriate antigen retrieval technique, such as microwave antigen retrieval or porteolytic digestion. Proteolytic antigen retrieval can be done using trypsin and pronase. Heat mediated antigen retrieval may be used in many cases to enable staining of certain antigens in paraffin embedded tissue sections, or in some cases to improve results when staining is weak or inconsistent. ${ }^{13,14}$

A number of buffers may be used for this purpose. Solutions used as buffers could be Tris buffered saline, $0.01 \mathrm{M}$ citrate buffer, $0.001 \mathrm{M}$ EDTA buffer for antigen retrieval. ${ }^{13}$

The fluorescence distribution and pattern found in our study was comparable with the previously reported studies on not only with paraffin embedded sections but also with fresh frozen sections. Hence, this technique, can be applied successfully to formalin fixed material. This implies that immunofluorescent study need not always require cryostat and frozen sections, but can be done in routine lab set up using paraffin embedded tissue blocks.

Universal diagnostic criteria with the help of molecular or immunologic studies on lichen planus is necessary. Also, it is important to distinguish it from lichenoid reaction and lichenoid dysplasia as all three of these have overlapping features and have serious premalignant potential. ${ }^{15}$

\section{CONCLUSION}

The diagnosis and immunopathogenesis of some oral mucosal diseases can be facilitated by the use of direct immunofluorescence study. Fresh frozen tissue is preferred when using this technique, however, frequently the material received by the histopathology laboratory has been fixed in formalin.

This particular study demonstrates the technique of direct immunofluorescence on paraffin embedded sections of oral lichen planus.

Out of 35 cases studied 34 showed fibrinogen deposition at basement membrane zone and 30 cases showed fibrin at small blood vessel wall along with basement membrane zone. 
The fluorescence distribution and pattern found in our study was comparable with the previously reported studies not only with paraffin embedded sections but also with fresh frozen sections.

Hence direct immunofluorescence study on paraffin embedded sections, proves to be a valuable diagnostic tool that improves the often controversial histopathological assessment of oral lichen planus.

\section{CLINICAL SIGNIFICANCE}

This study provides a reliable method which could be used for diagnostic purposes in instances where formalin fixed tissue only will be received for histopathologic evaluation and where the laboratory lacks expensive armamentarium like cryostat.

\section{REFERENCES}

1. Laskaris G, Sklavounou A, Angelopoulos A. Direct immunofluorescence in oral lichen planus. Oral Surg Oral Med Oral Pathol 1982 May;53(5):483-487.

2. Kolde G, Wesendahl C, Stein H, Reichart PA. Oral lichen planus: diagnostic immunofluorescence testing on routine histological material. Br J Dermatol 2003 Feb;148(2):374-376.

3. Firth NA, Rich AM, Radden BG, Reade PC. Direct immunofluorescence of oral mucosal biopsies: a comparison of fresh-frozen tissue and formalin-fixed, paraffin-embedded tissue. J Oral Pathol Med 1992 Sep;21(8):358-363.

4. Firth NA, Rich AM, Radden BG, Reade PC. Assessment of the value of immunofluorescence microscopy in the diagnosis of oral mucosal lichen planus. J Oral Pathol Med 1990 Aug; 19(7):295-297.

5. Raghu AR, Rao NN. Immunofluorescence in oral lichen planus and oral lichenoid reaction: a review. Ind J Dent Res 2001 Jan-Mar;12(1):29-34.

6. Helander SD, Rogers RS. III, The sensitivity and specificity of direct immunofluorescence testing in disorders of mucous membranes. J Am Acad Dermatol 1994 Jan;30(1):65-75.

7. Walsh LJ, Savage NW, Ishii T, Seymour GJ. Immunopathogenesis of oral lichen planus. J Oral Pathol Med 1990;19: 389-396.

8. Vinay K, Ramzi CS, Stanley RL. Robbins basic pathology. 7th ed; Elsevier Publishers, 2003. p. 119-120.

9. Abell E, Presbury DG, Marks R, Ramnarain D. The diagnostic significance of immunoglobulin and fibrin deposition in lichen planus. Br J Dermatol 1975 Jul;93(1):17-24.

10. Daniels TE, Quadra-White C. Direct immunofluorescence in oral mucosal disease: a diagnostic analysis of 130 cases. Oral Surg Oral Med Oral Pathol 1981 Jan;51(1):38-47.

11. Nieboer C. The reliability of immunofluorescence and histopathology in the diagnosis of discoid lupus erythematosus and lichen planus. Br J Dermatol 1987 Feb;116(2):189-198.

12. Raghu AR, Nirmala NR, Sreekumaran N. Direct immunofluorescence in oral lichen planus and oral lichenoid reactions. Quintessence Int 2002 Mar;33(3):234-239.

13. Antigen retrieval techniques for use with formalin fixed Paraffin Embedded Sections. www.serotech.com

14. Shi SR, Cote RJ, Taylor CR. Antigen retrieval techniques: current perspectives. J Histochem Cytochem 2001 Aug; 49(8):931-937.

15. Patil S, Rao RS, Sanketh DS, Sarode SC, Sarode GS. A universal diagnostic criteria for oral lichen planus: an exigency! Int J Contemp Dent Med Rev Vol. 2014. Article ID 041214, 2014. 